\title{
Percutaneous vertebroplasty at C7 for the treatment of painful metastases -A case report-
}

\author{
Sung-Suk Seo ${ }^{1}$, Dong-Heon Lee ${ }^{2}$, Hae-Jin Kim ${ }^{1}$, Ji-Wook Yoon ${ }^{1}$, Oh-Sun Kwon ${ }^{1}$, and Kyung-Hoon Kim ${ }^{1}$ \\ ${ }^{1}$ Department of Anesthesia and Pain Medicine, School of Medicine, Pusan National University, Yangsan, ${ }^{2}$ Department of \\ Anesthesiology and Pain Medicine, Chnagwon Samsung Medical Center, Changwon, Korea
}

The cervical spine is a less common site for metastatic disease than is the thoracolumbar spine. Percutaneous vertebroplasty (PVP) in the cervical spine can be performed using an anteromedial or lateral approach. A 51-yearold woman with breast cancer had been experiencing severe weight-bearing neck pain for 2 months, even after undergoing radiation therapy. Imaging studies revealed an osteolytic compression fracture in the C7 vertebra. For performing PVP at C7 using the anteromedial approach, a needle was inserted from the left side of the patient's neck. The needle was advanced to the anterior $1 / 3$ anterior $1 / 3$ or $1 / 2$ of the vertebral body by hammering, and approximately $2 \mathrm{ml}$ of cement was injected. Immediately after the operation, the patient could move her neck without pain. In conclusion, PVP using an anterolateral approach may be an option for treating metastatic osteolytic vertebral lesions in the cervical spine for alleviating intractable axial neck pain. (Korean J Anesthesiol 2013; 64: 276-279)

Key Words: Breast neoplasm, Cervical vertebrae, Neoplasm metastasis, Pain, Palliative care, Vertebroplasty.

Bone is the most frequent metastatic location for breast cancer; however, the occurrence of a single cervical metastasis in breast cancer is rare. The cervical spine is a lesser common site of metastatic disease (10\%) than is the thoracic or lumbar spine ( $70 \%$ or $20 \%$, respectively). Approximately $50 \%$ of spinal metastases arise from 1 of 3 primary types of cancer: breast, lung, or prostate [1].

Percutaneous vertebroplasty (PVP) is performed using a transpedicular or extrapedicular approach in the thoracic or lumbar spine; however, it can be performed using an anterolateral or posterolateral approach in the cervical spine. Pain specialists are accustomed to using the same anteromedial approach for the cervical discogram or percutaneous endoscopic cervical discectomy. A right-handed operator pushing the tracheo-esophageal complex toward the opposite side with the left index and middle fingers and then inserts these fingers inside towards the front of the vertebral body [2].

We have previously reported a case of PVP at C2 using an

Received: January 27, 2012. Revised: 1st, March 8, 2012; 2nd, April 24, 2012. Accepted: May 23, 2012.

Corresponding author: Kyung-Hoon Kim, M.D., Department of Anesthesia and Pain Medicine, School of Medicine, Yangsan Busan National University Hospital, Beomeo-ri, Mulgeum-eup, Yangsan 626-770, Korea. Tel: 82-55-360-1422, Fax: 82-55-360-2129, E-mail: pain@pusan.ac.kr (c) This is an open-access article distributed under the terms of the Creative Commons Attribution Non-Commercial License (http:// creativecommons.org/licenses/by-nc/3.0/), which permits unrestricted non-commercial use, distribution, and reproduction in any medium, provided the original work is properly cited. 
anterolateral approach [3]; however, there are few reported cases of PVPs performed in metastatic C3-C7 vertebrae because of their limited weight-bearing nature and the rarity of a single metastatic cervical vertebral body [4]. Herein we report a successful case of PVP at C7 using the anterolateral approach for the treatment of painful metastasis.

\section{Case Report}

A 51-year-old woman with breast cancer had been experiencing severe neck pain for 2 months. She felt severe weightbearing pain in her neck, and occasionally radiating to her entire right arm, even after undergoing radiation therapy. Imaging studies revealed an osteolytic compression fracture in the $\mathrm{C} 7$ vertebra (Fig. 1).

The patient refused to undergo open corpectomy with fusion; therefore, we decided to perform PVP at C7. Permission for a clinical trial was granted by the Institutional Review Board from the hospital, and informed consent about potential complications, including nerve injury, bleeding, tracheoesophageal injury, wound infection, and embolism, was obtained from the patient. The patient received $1 \mathrm{~g}$ of cefazolin intravenously $30 \mathrm{~min}$ before the operation. The procedure was performed under conscious sedation with basic monitoring such as electrocardiography, pulse oximetry, and noninvasive monitoring of blood pressure. The patient was placed in the supine position on a radiolucent table with an inflatable, adjustable pillow placed behind her neck. After aseptic draping, local anesthesia was provided with a $5 \mathrm{ml}$ of $1 \%$ lidocaine and intravenous analgesia with $30 \mathrm{mg}$ of ketorolac and $100 \mu \mathrm{g}$ of fentanyl.

A right-handed operator pushed tracheo-esophageal complex toward the opposite side with the left index and middle fingers; subsequently the operator inserted these fingers inside towards the front of the vertebral body [2]. An additional $2 \mathrm{ml}$ of $1 \%$ lidocaine was administered on the anterior longitudinal ligament and periosteum of the anterior $\mathrm{C} 7$ vertebral body. A 13 gauge, $10 \mathrm{~cm}$ long bone biopsy needle was inserted into
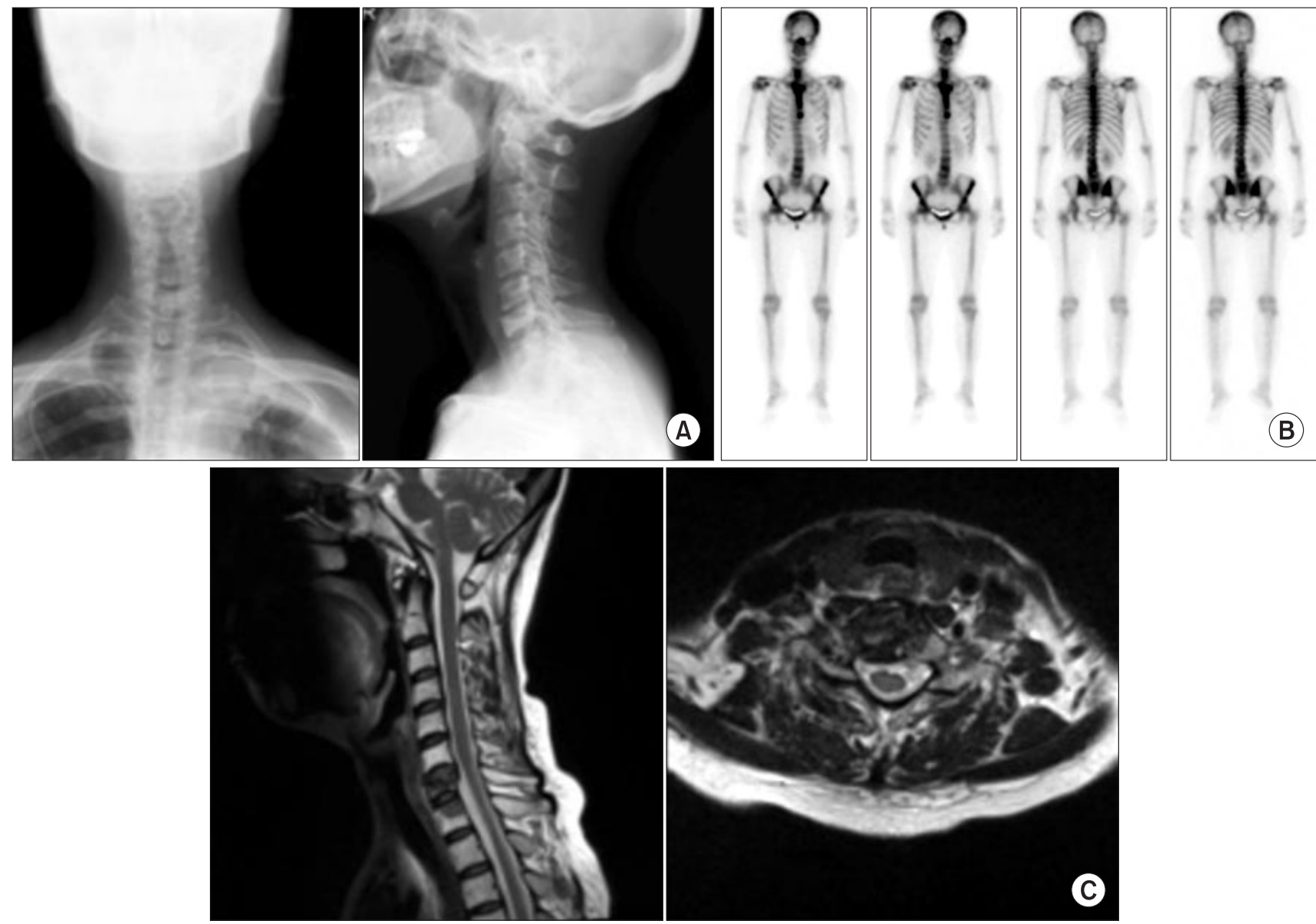

Fig. 1. Preoperative images revealed an osteolytic compression fracture at C7. (A) Anteroposterior and lateral plain films. (B) Bone scans at C7. (C) Magnetic resonance imaging. 
the left side of the patient's neck. The needle was advanced into the anterior $1 / 3$ anterior $1 / 3$ or $1 / 2$ of the vertebral body by hammering, which was necessary because of the hardness of the cortex of the vertebral body. After confirming needle placement and ensuring that no contrast medium was leaking into the vessels or epidural space, $2 \mathrm{ml}$ of cement was injected (Fig. 2).

Immediately after the operation, the patient could stand and sit without neck pain. There were no complications, such as bleeding, vagus nerve injury, tracheo-esophageal injury, cement leakage or embolism. Fortunately, no evidence of new metastases was found and her neck pain was gone completely during the 6 month follow-up.

\section{Discussion}

From a superior view of the transverse section of through the neck at the level of the $\mathrm{C} 7$ vertebra, distinct anterior and posterior components can be identified. The anterior portion is surrounded by the cervical fascia, which consists of the pretracheal, superficial, and prevertebral layers. A visceral fascia encloses the tracheo-esophageal complex and left and right thyroid glands. The right and left carotid sheathes enclose the common carotid artery, internal jugular vein, and vagus nerve.
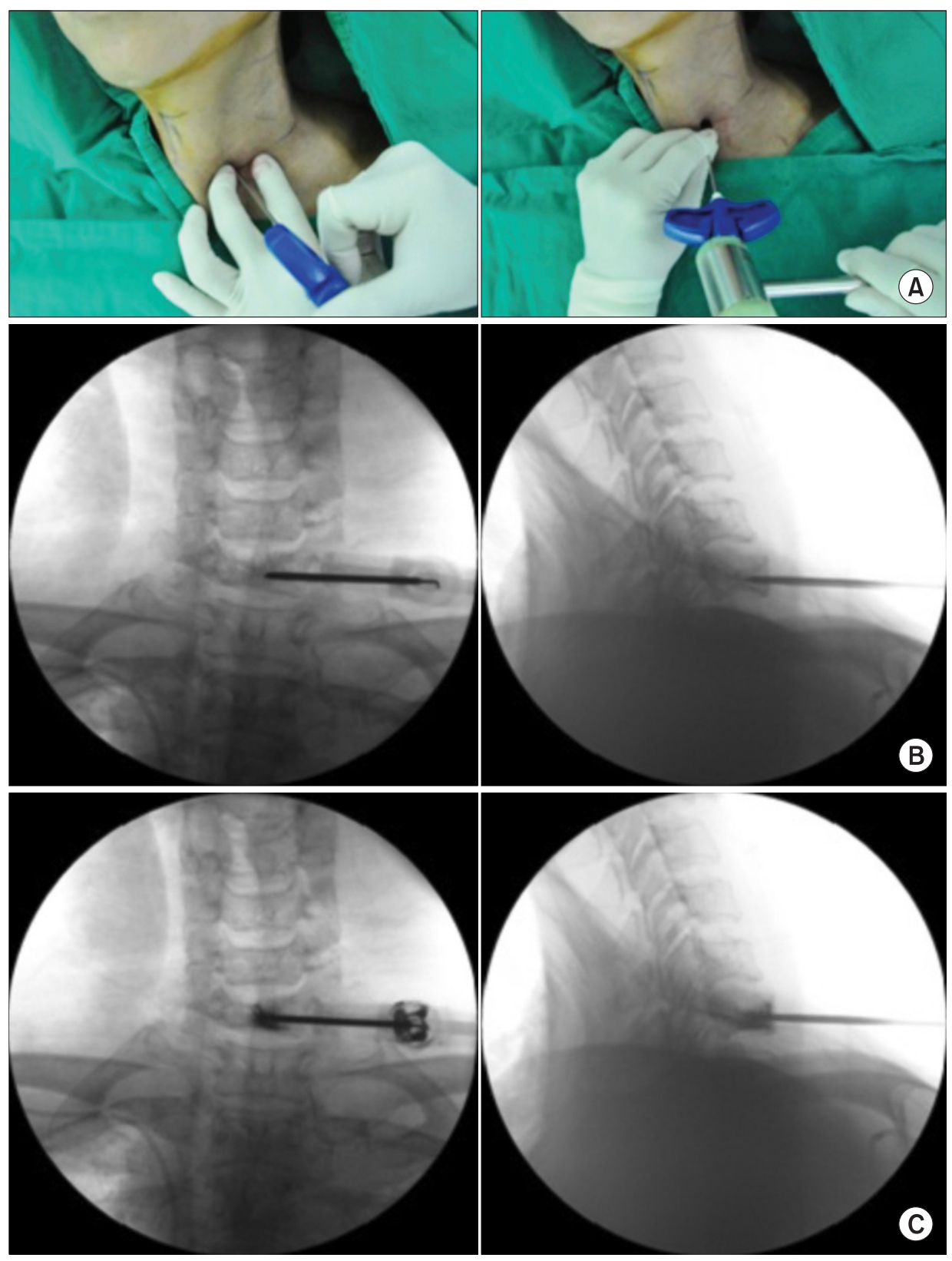

Fig. 2. Percutaneous vertebroplasty at C7. (A) Photographs of the operation filed. (Left) A right-handed operator pushed the tracheo-esophageal complex toward the opposite side with the left index and middle fingers, and then inserted these fingers inside towards the front of the vertebral body. A 13-gauge, $10-\mathrm{cm}$ long bone biopsy needle was inserted into the left side of patient's neck. (Right) The needle was advanced into the targeted anterior $1 / 2$ or $1 / 3$ of the vertebral body by hammering because of the hardness of the cortex of the vertebral body. (B) After confirming needle placement and ensuring that no contrast medium had leaked into the vessels or epidural space, (C) $2 \mathrm{ml}$ of cement was injected. 
The posterior portion is surrounded by the nuchal fascia, which consists of the superficial and deep layers.

Performing PVPs in the cervical vertebrae is usually done via an anterolateral approach; however, PVPs in the thoracic or lumbar vertebrae is commonly used via a transpedicular approach. It is necessary to confirm that the needle does not penetrate major anatomical structures, such as the tracheoesophageal complex, carotid artery, internal jugular vein, or vagus nerve. To avoid serious complications of penetrating the above major organs, a right-handed operator pushed tracheoesophageal complex toward the opposite side with the left index and middle fingers. Subsequently, the operator inserts these fingers inside towards the front of the vertebral body while feeling the pulsation of the carotid artery with the radial side of the abducted index finger. And then, the needle must be placed between the fingers. After the needle contacts the anterior vertebral body via the anterior longitudinal ligament, anchoring the needle by hammering is a challenging procedure owing to the hardness of the cortex. However, after the needle penetrates the cortex, further advancement of the needle to the targeted anterior $1 / 3$ or $1 / 2$ of the vertebral body, depending on the shape of the fracture, is feasible without hammering.

Recently, a useful case review article has reported a relative large number of PVP cases in the cervical spine. This article reviewed 35 cases of PVP performed at $\mathrm{C} 1$ and $\mathrm{C} 2$ using the transoral approach, and 35 cases of PVP performed from C3 to C7 using the anterolateral or posterolateral approach among 62 patients. PVP eligibility criteria in these studies included: (1) vertebral fracture resulting from osteolytic metastasis in the cervical spine with integrity of the posterior vertebral wall; (2) severe debilitating pain or loss of mobility that could not be relieved by appropriate oncologic or medical therapy; (3) failed radiation therapy or chemotherapy with a life expectancy of between 3 and 6 months; (4) and/or the presence of comorbidities contraindicating surgery [5]. Painful metastatic lytic lesions of the cervical spine could be also treated by percutaneous anterolateral balloon kyphoplasty in case of disruption of the posterior vertebral wall $[4,6]$.

The spinal column is the most common location among osseous sites for metastatic deposits. Spinal involvement may occur in up to $40 \%$ of patients with cancer. However, spinal cord compression from epidural metastases occurs in only $5-10 \%$ of cancer patients. The posterior body of the vertebral body is usually involved first, with subsequently involvement of the anterior body, lamina, and pedicles [1].

Generally, excisional surgery is only performed for patients with an estimated life expectancy of greater than 3 months [1]. In an effort to more accurately predict survival, Tokuhashi et al. [7] proposed a preoperative prognostic scoring system. According to their scoring system, the patient received a total score of 15 (general condition, 2; number of extraspinal bone metastases foci, 2; number of metastases in the vertebral body, 2; metastases to the major internal organs, 2; primary site of the cancer, 5; and palsy, 2) had an average survival of 12 months or more. PVP using polymethylmethacrylate (PMMA), although considered a palliative treatment, has a potential ability of the exothermic effect during polymerization, which may contribute to tumor necrosis and reduction in tumor volume. When unpolymerized, PMMA is a strong cytotoxic agent with destructive activity against neoplastic cells [8].

In conclusion, we report a successful case of PVP at C7 using the anterolateral approach for the treatment of painful metastasis under local anesthesia and intravenous analgesia without complications. However, continuous follow-up is required for detecting recurrence at the same site, invasion of different level of the spine, or extraspinal metastases.

\section{Acknowledgments}

This study was supported by a 2-year research grant from Pusan National University.

\section{References}

1. Klimo P Jr, Schmidt MH. Surgical management of spinal metastases. Oncologist 2004; 9: 188-96.

2. Ahn Y, Lee SH, Shin SW. Percutaneous endoscopic cervical discectomy: clinical outcome and radiographic changes. Photomed Laser Surg 2005; 23: 362-8.

3. Yoon JY, Kim TK, Kim KH. Anterolateral percutaneous vertebroplasty at $\mathrm{C} 2$ for lung cancer metastasis and upper cervical facet joint block. Clin J Pain 2008; 24: 641-6.

4. Druschel C, Schaser KD, Melcher I, Haas NP, Disch AC. Minimally invasive combined anterior kyphoplasty for osteolytic C2 and C5 metastases. Arch Orthop Trauma Surg 2011; 131: 977-81.

5. Masala S, Anselmetti GC, Muto M, Mammucari M, Volpi T, Simonetti G. Percutaneous vertebroplasty relieves pain in metastatic cervical fractures. Clin Orthop Relat Res 2011; 469: 715-22.

6. Lykomitros V, Anagnostidis KS, Alzeer Z, Kapetanos GA. Percutaneous anterolateral balloon kyphoplasty for metastatic lytic lesions of the cervical spine. Eur Spine J 2010; 19: 1948-52.

7. Tokuhashi Y, Ajiro Y, Umezawa N. Outcome of treatment for spinal metastases using scoring system for preoperative evaluation of prognosis. Spine (Phila Pa 1976) 2009; 34: 69-73.

8. Mikami Y, Numaguchi Y, Kobayashi N, Fuwa S, Hoshikawa Y, Saida Y. Therapeutic effects of percutaneous vertebroplasty for vertebral metastases. Jpn J Radiol 2011; 29: 202-6. 